\title{
Precision Arrival Scheduling for Tactical Reconfiguration
}

\author{
Shannon Zelinski* \\ NASA Ames Research Center, Moffett Field, CA, 94035
}

\begin{abstract}
This research adapts the concept of precision arrival scheduling to accommodate reconfiguration operations between two sets of fixed arrival routes modeled for Chicago O'Hare International Airport (ORD). Integrated fixed path routing from en-route to runways was modeled for ORD's top two peak traffic configurations, as well as transition routing between the two configurations. A first-come-first-served multi-point scheduler was adapted to the reconfiguration problem by prioritizing the rescheduling of aircraft within the terminal airspace at the time of reconfiguration notification. Arrival rescheduling was then tested for a range of arrival rates and reconfiguration notification lead-times in fasttime simulation. Reconfigurations with lead-times as short as 10 minutes at a nominal static configuration arrival rate ( $\sim 25$ arrivals per quarter hour) could be accommodated with little impact to throughput. However, as lead-time shortened below 25 minutes, individual aircraft efficiency quickly degraded due to extra flight time at lower altitude and speed. In general, first-come-first-served arrival scheduling on fixed routing for this sample reconfiguration problem is promising if at least $\mathbf{1 0 - 1 5}$ minutes lead-time is given before the reconfiguration is in effect.
\end{abstract}

\section{Nomenclature}

$=$ flight

$=$ node

$=$ required separation distance

$=$ time

$=$ notification time

$=$ reconfiguration time

$=$ nominal speed

\section{Introduction}

$\mathrm{R}$ ECONFIGURATION is a change in airport runway usage, usually referring to a change in airspace flow direction as well. There are many reasons why an airport may reconfigure: a change in noise restrictions between day and night, a shift in demand between arrivals and departure or between dominant flow directions, or a change in wind direction or magnitude making it difficult to land in the current configuration. A nation-wide analysis of reconfiguration events showed that reconfiguration can have a significant impact on airport capacity causing loss of throughput at larger busier airports. ${ }^{1}$ More detailed analyses of Dallas/Fort Worth and Chicago O'Hare (ORD) reconfiguration operations distinguished between strategic and tactical reconfigurations in how delay is absorbed. ${ }^{2,3}$ Strategic reconfigurations are planned and coordinated by the traffic managers at the Tower, Terminal Radar Approach Control (TRACON), and Center levels. They are proactive in nature and most of the delay is absorbed outside of the TRACON. By contrast, tactical flow changes occur with minimal warning to or planning by the traffic managers. They are reactive in nature and delay is absorbed both inside and outside the TRACON. Whereas strategic reconfigurations are generally more common than tactical, tactical reconfigurations are more disruptive, especially in high traffic load conditions. Ref. 3 studied seven cases of reconfiguration between the top two ORD configurations occurring in April 2012, three of which resulted in disruptive holding and vectoring both inside and outside the TRACON.

Prior research has focused on the more strategic problem of optimally scheduling the reconfiguration event itself with a 90 -minute or greater planning horizon. ${ }^{4-6}$ Reference 7 briefly addresses the problem of scheduling individual flights given a planned reconfiguration time. However, flights are scheduled to runways assuming the

\footnotetext{
${ }^{*}$ Senior Research Engineer, Aerospace High Density Operations Branch, Mail Stop 201-06, AIAA Senior Member
} 
reconfiguration time is known hours in advance, falling into the strategic reconfiguration category. No scheduling research could be found in the current literature specifically addressing tactical reconfiguration.

This research addresses the tactical reconfiguration scheduling problem with the concept of precision arrival scheduling along fixed routing. In nominal conditions, precision arrival scheduling concepts can increase peak airport throughput $10 \%{ }^{8}$ These concepts use fixed routing defined from the meter fix all the way to the runway to more accurately estimate time-to-fly, enabling more precise scheduling with smaller buffers, yielding higher throughput. However, these concepts must be made robust to off-nominal disturbances. Whereas research has begun to explore and enhance precision scheduling robustness to disturbances such as wind errors, emergency landings, and missed-approaches, ${ }^{9,10}$ these were all tested with static arrival fixed routing. In the case of airport reconfiguration, fixed routing may no longer be fixed as one set of routes is swapped out for another. This paper extends the concept of precision arrival scheduling to this dynamic reconfiguration environment.

Integrated fixed arrival routing is modeled for the top two peak traffic configurations at ORD. A first-come-firstserved multi-point scheduler is adapted to the tactical reconfiguration problem by rescheduling aircraft within the TRACON upon reconfiguration notification. Then the effect of the rescheduling on throughput and delay metrics is studied for a range of arrival rates and reconfiguration notification lead-times.

\section{Method}

\section{A. Route Models}

An analysis of 2011 and 2012 ORD reconfiguration operations ${ }^{3}$ determined that the most common reconfiguration changes occurred between the two most commonly used arrival configurations. These are Plan X Trip (PX) using arrival runways $10,09 \mathrm{R}$ and $04 \mathrm{R}$, and West Flow (WF) using arrival runways $27 \mathrm{~L}$, 27R and $28^{\dagger}$ depicted in Figure 1.

Arrival route models for these configurations were generated using published routes and historical flight track data. Published routes were extracted from all ORD Standard Terminal Arrival Routes (STARs) and Instrument Approach Procedures (IAPs) for the six runways used by the PX and

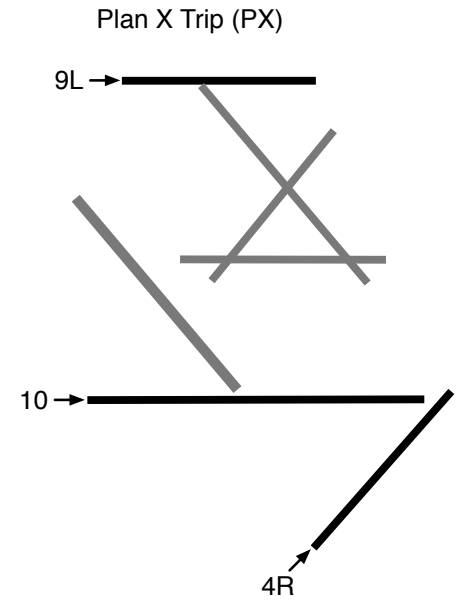

Figure 1. Top two ORD arrival runway configurations. WF configurations."

Daily operating conditions by the quarter-hour for ORD for the entire year of 2012 were collected from Aircraft System Performance Metrics (ASPM) Airport Efficiency reports. ${ }^{11}$ Among other data, these reports included a list of runways configured for arrivals, the arrival rate capacity, and the actual number of arrival operations at ORD every quarter-hour. ORD was assumed to be in PX or WF configuration for a given quarter hour if all of their respective arrival runways were listed as configured for arrivals in the ASPM report. A total of 34 24-hour historic traffic samples were selected from 2012 based on their relatively high number of actual arrival operations during PX and WF configurations. Historical track data were collected for all aircraft that arrived at one of the PX or WF configuration runways during a quarter-hour when ORD was in that configuration. A total of 29,660 flight tracks were collected, roughly half landing in each configuration.

Figure 2 shows jet tracks, which comprised $99.3 \%$ of the tracks collected, and the published routes that most closely represent them. The concentric circles are centered on the ORD fix with radii increasing in 10 mile increments to 100 miles. Tracks of flights landing in PX and WF configurations are shown in gray and $\tan (\tan$ overlaying gray), respectively. STAR common and transition routes are overlaid in blue and maroon respectively. IAPs for the six runways of interest are shown in green. Whereas STARs (common and transition) capture much of

\footnotetext{
${ }^{\dagger}$ In late 2013, new runways were added to ORD and some runway designations have changed.

* STARs and IAPs were extracted from En Route Automation Modernization (ERAM) adaptation data (http://www.faa.gov/air_traffic/technology/eram/) accessed in July, 2013.
} 
the track paths, there are no transition paths defined between STARs and IAPs. In addition, several flows from the North do not follow any published STAR. Fixes MSN and BJB identify likely coordination points for these flows.

For the purposes of studying configuration changes, the route model should have entry points that both configurations use with similar frequency so that comparisons can be made using the same traffic scenarios for both configurations. For the Northeast, Northwest, and Southwest flows to meet this criterion, it would be sufficient for the model to extend roughly 45 miles from ORD at the beginning of the STAR common routes where the handoff between Center airspace and TRACON airspace typically occurs. However, the North flow undefined by STARs extends more than 80 miles from ORD before a common entry point (BJB) is found. The two STAR common routes serving the Southeast flows extend much farther from ORD (more than 90 miles) than the other STAR common routes. In addition, the more southern route (ROYKO) is more commonly used in PX and the more northern route (WATSN) is more commonly used in WF. Therefore, the route model was extended to entry points roughly 80-90 miles from ORD (roughly 40-50 miles from the TRACON).

Because the Northwest and Northeast sets of flight tracks each clearly merge into a single common route near the Center/TRACON handoff point, a single transition route entry point was chosen to represent each set of merging flows. These were MYTCH in the Northwest and WLTER in the Northeast. This assumes that if a schedule is given 110-160 miles from the TRACON, then by the time flights are at these entry points roughly 40-50 miles from the TRACON, the Center controller has arranged flights in the correct sequence and spacing for the merge.

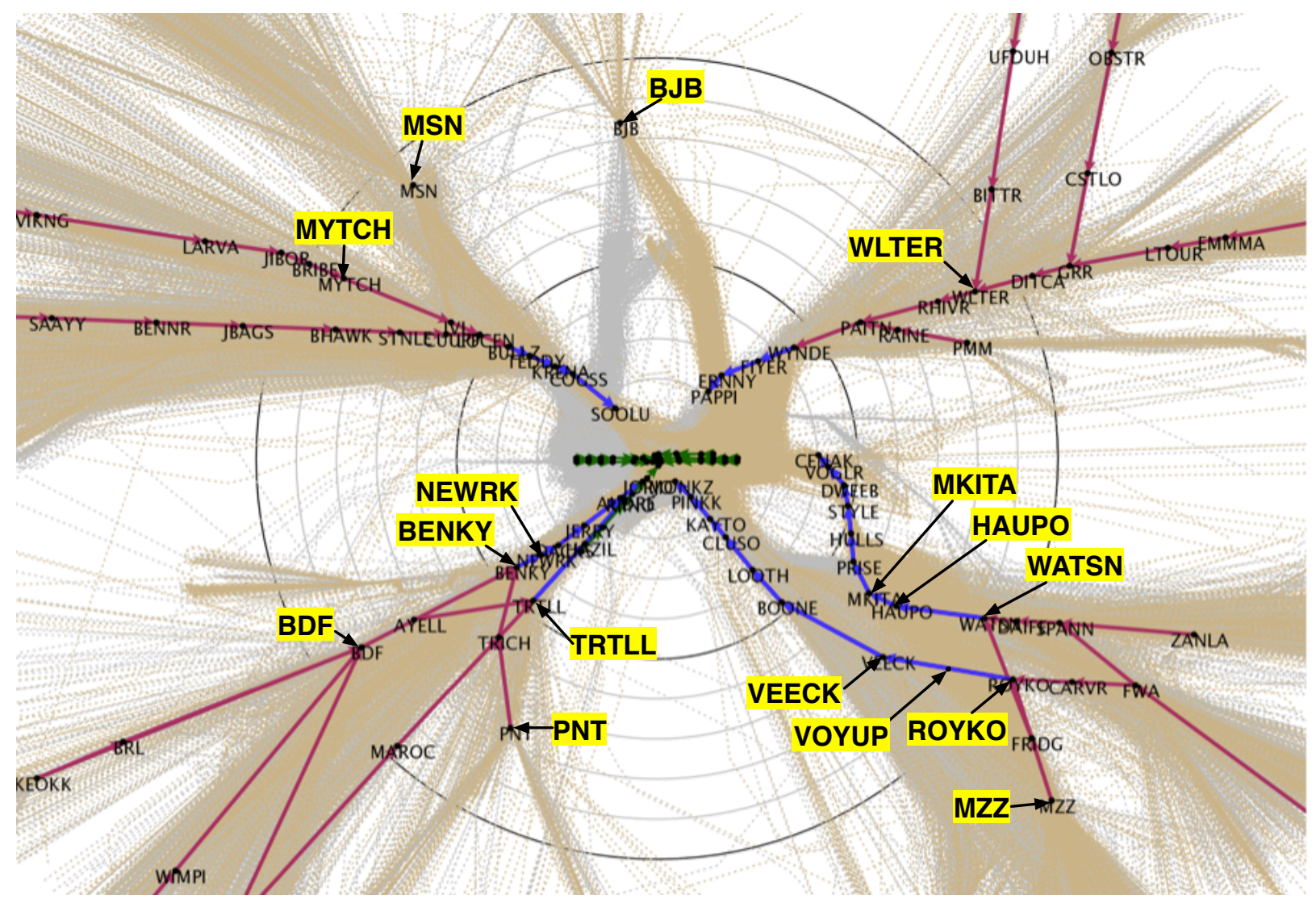

Figure 2. Flight tracks and published STARs and IAPs.

It is less clear by looking at the raw flight tracks how simplified transition routing should be modeled to represent Southwest and Southeast flows because they each feed two STAR common routes. In order to inform the design of simplified transition route modeling in the Southwest and Southeast and original transition routing in the North, historical flight tracks were processed ${ }^{12}$ to identify the most commonly used flight paths. Flight tracks for jets were processed separately for each arrival runway with a clustering parameter that separated nodes in the resulting node-link route model by at least 3 miles.

Analysis of the Northwest and Northeast clustered flight paths verified that these flows merged near their respective common routes. Analysis of the Northern flows also verified BJB as best entry point common to both 
configurations. Although a second common entry exists originating from General Mitchell International Airport 55 miles North of ORD, this entry was not modeled, as these flights are relatively infrequent and too far within the 150200 mile arrival scheduling freeze horizon. Future research should address integration of arrival traffic from nearby satellite airports.

Analysis of the Southeast clustered flight paths revealed that there are two distinct sets of merging flows that tend to feed the WATSN common route in $\mathrm{WF}$ and the ROYKO common route in PX. One flow merges near MZZ and then diverges to either VEECK or HAUPO. The other flow merges at WATSN and then either continues along the WATSN common route or diverges at MKITA. To model this crossover, WATSN and VOYUP were modeled as distinct Southeast entry points. VOYUP also served as the diverging point between VEECK and HAUPO for the MZZ flow.

Analysis of the Southwest clustered flight paths revealed that whereas two distinct flows exist at BDF and PNT, they both feed primarily the BENKY common route. Even most PNT traffic landing on 04R, whose IAP overlaps the TRTLL common route, merge with the BENKY common route at NEWRK before turning back to the 04R IAP. Therefore, BDF was modeled as a single entry point representing all Southwest flows.

To aid more detailed route modeling within the TRACON, track data were processed ${ }^{12}$ to identify the most commonly used flight paths again, this time with a clustering parameter that separated nodes by at least 1.5 miles. Once again, Jets were processed separately for each arrival runway. Once all lateral paths had been designed, flight track altitudes along crossing paths were analyzed to determine if altitude separation is maintained. If nominal altitude ranges overlapped between crossing paths, a shared intersection node was added to ensure lateral separation through scheduling.

Figure 3 shows the integrated route model including multiple commonly used path options from each of six entry nodes to each of the six runways

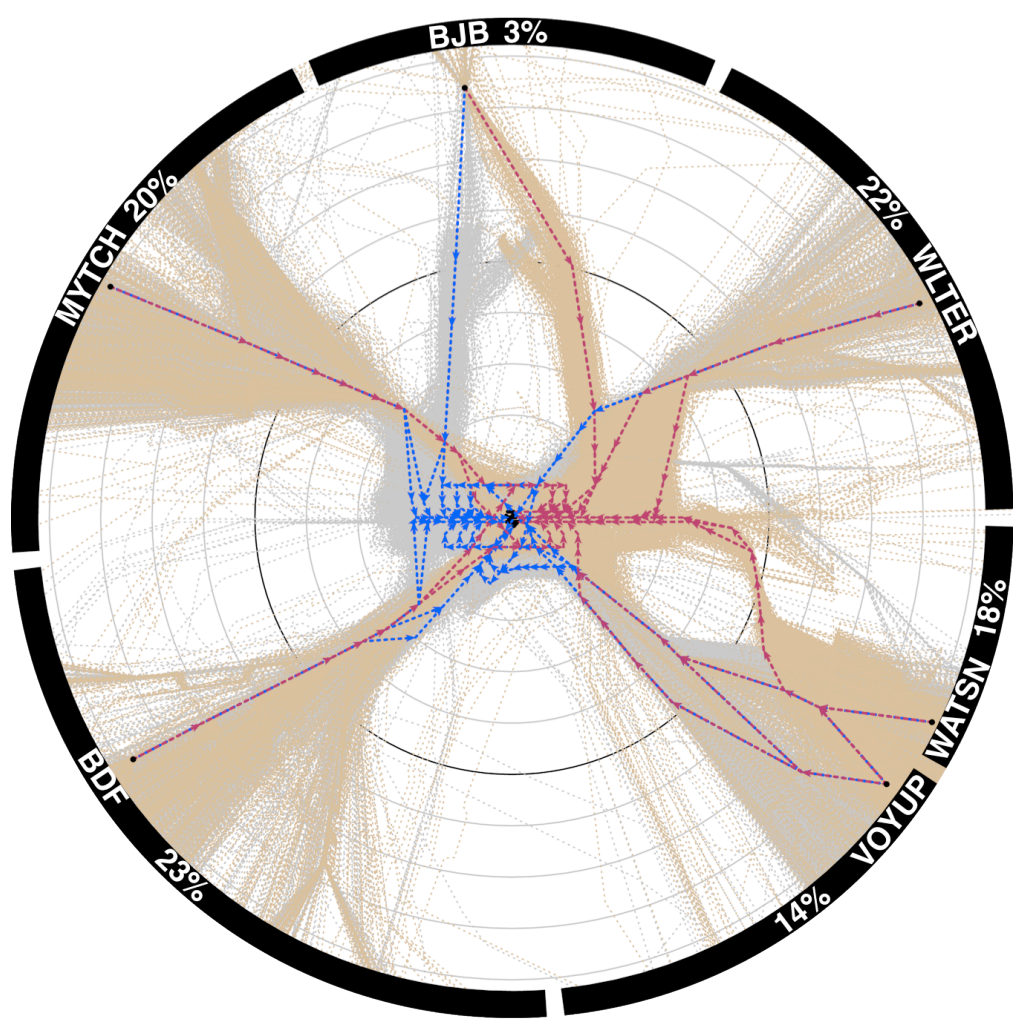

Figure 3. Integrated route model.

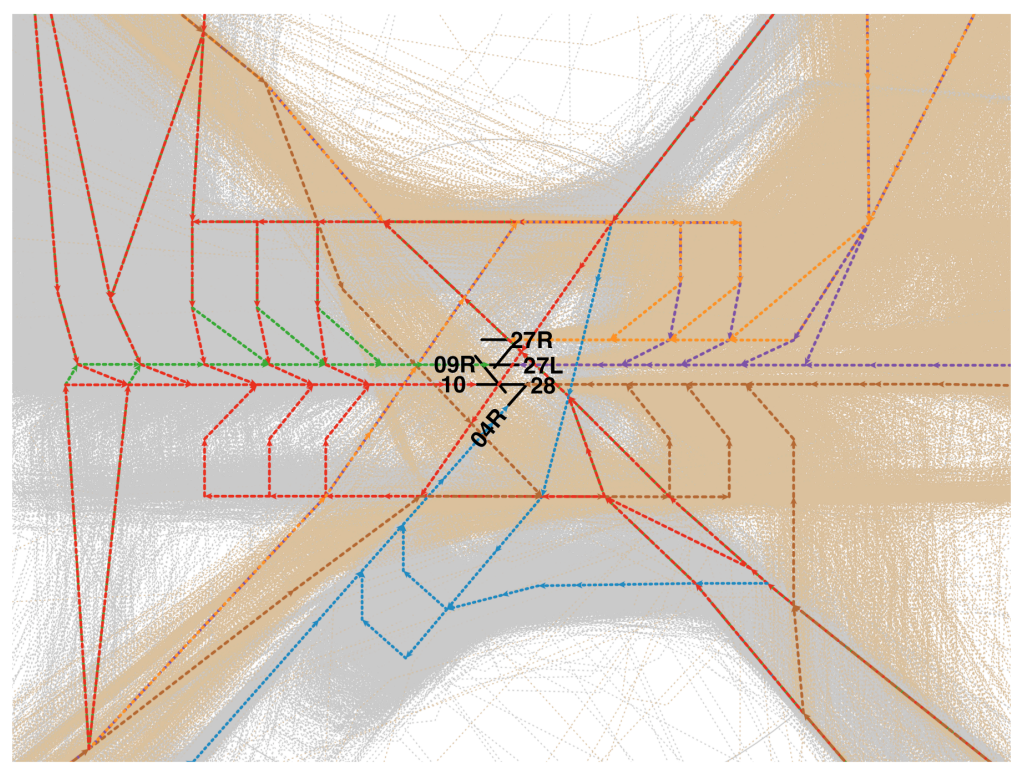

Figure 4. Integrated route model TRACON detail.

modeled. Routes serving PX (blue) and WF (maroon) runways are shown overlaying historical flights tracks (gray and tan, respectively) to those runways. Thick black arcs identify the arc range and percentage of arriving traffic that 
each modeled entry point represents. Percentages are based on each flight's first track point within 90 miles of ORD. Figure 4 shows the integrated route model magnified to show detailed routing in the TRACON close to the runways. Routes are color coded by the runways they serve. Unlike current precision arrival schedulers, which use a single path option per arrival fix-runway pair for a given aircraft, notice that multiple path options to each runway are available to the arrival scheduler including base leg extensions and crossovers. These path options give the scheduler additional control authority beyond speed alone.

In addition to the static configuration routes, transition routes between static configuration routings were generated to ensure a path existed from every node of one configuration to at least one runway of

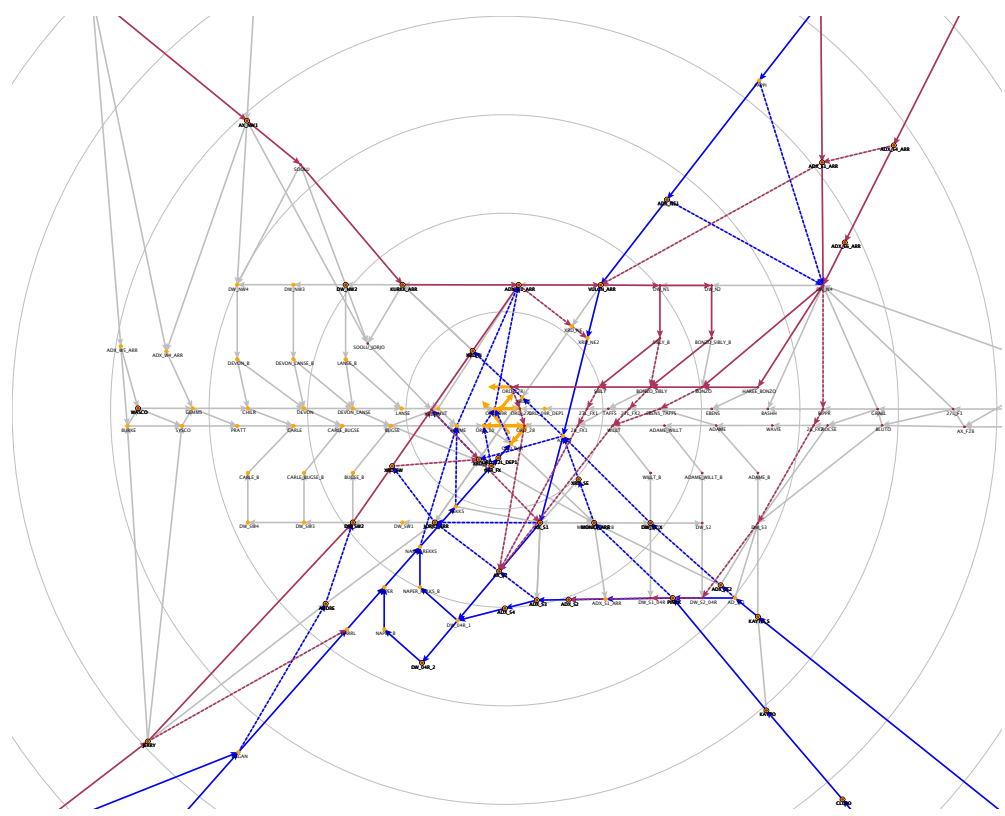

Figure 5. Transition routes designed between $04 R$ and $27 R$.

the other configuration. This included runway sink nodes to test the case of reconfiguration without pre-notification. Transition routes were designed to rejoin static routing as soon as reasonable with as few intersections and new nodes as possible. Figure 5 shows transition routes designed between 04R (blue) and 27R (maroon).

\section{B. Arrival Scheduler}

A first-come-first-served multi-point scheduling technique ${ }^{13}$ was applied to the route model. The scheduler was implemented in fast-time in the Python programming language. Flights were scheduled in order of their Estimated Time of Arrival (ETA) at their entry node to emulate distance-based first-come-first-served ordering. As each new flight is considered, the scheduling technique produces a sequence of feasible time windows for which the flight may be scheduled at each node along a given route. The time windows must satisfy feasible ranges of transit time along each route segment and avoid blocked time slots at each node reserved for the Scheduled Times of Arrival (STAs) of previously scheduled flights. Then the earliest arrival times available at each node, especially the runway, are considered the preferable schedule for that route. Because the route model includes not only multiple arrival runway options, but also multiple route options to each runway, a schedule was produced for each route option to each runway and the one providing the earliest runway arrival time was chosen. The assigned route option with the earliest runway STA may not necessarily be the one with the earliest runway ETA.

\section{Transit time ranges}

Transit time ranges were used to implicitly model how much delay could be absorbed along a given route segment with speed control. Because historical flight tracks may include path stretch delay, the nominal transit time along a route segment between two nodes was calculated based on nominal speeds observed in historical track data at end nodes and segment path distance. For each historical flight, the closest track point within 3 miles of each node was collected and analyzed to assign nominal speeds to the node. The median speeds for all flights landing at the same runway tended to differ slightly for some nodes if the flight path distance from the node to the runway was significantly different. Therefore, nodes were assigned separate nominal speeds for PX and WF, and in some cases a separate 04R speed from the other PX runways was assigned as well.

Assume a flight plans to travel a route segment from node $n_{1}$ to node $n_{2}$ and the nominal speeds at $n_{1}$ and $n_{2}$ given its planned arrival runway are $v_{1}$ and $v_{2}$ respectively. The nominal transit time from $n_{1}$ to $n_{2}$ is calculated as: 


$$
t_{\text {nom }}\left(n_{1}, n_{2}\right)=\left\{\begin{array}{cc}
\frac{d\left(n_{1}, n_{2}\right)}{\left(v_{1}+v_{2}\right) / 2} & v_{1}>v_{2} \\
\frac{d\left(n_{1}, n_{2}\right)}{v_{1}} & v_{1} \leq v_{2}
\end{array},\right.
$$

where $d\left(n_{1}, n_{2}\right)$ is the distance between $n_{1}$ and $n_{2}$. Note that if nominal speed is decreasing $\left(v_{1}>v_{2}\right)$, transit time is distance divided by average node speed. Because arrival flights are not expected to accelerate (only decelerate), if $v_{1}$ $<v_{2}$, as can be the case along a reconfiguration transition route, the nominal transit time is distance divided by the slower nominal speed $v_{1}$. Just as a node's nominal speed may differ by runway, the route segment nominal transit time may differ by runway as well. The nominal transit time was considered to be the minimum (i.e. fastest) schedulable transit time, reserving faster performance to manage uncertainty when trying to meet the schedule. Thus, the scheduler may not time advance flights, only delay. Because it is estimated that speed control alone can modify flight time up to $10 \%$, the maximum (i.e. slowest) transit times were assumed to be $10 \%$ higher than the nominal transit times.

\section{Blocked time slots}

The size of a blocked time slot was used to implicitly model the radar separation minimum at a given node. Per FAA regulation, ${ }^{14}$ the separation minimum is 5 miles when the aircraft are 40 miles or more from the terminal radar antenna. The separation minimum is 3 miles when the aircraft are less than 40 miles from the antenna. Aircraft conducting an instrument approach must be separated using wake turbulence minima, which depends on the relative weight classes of aircraft pair being considered. Based on the weight class mix of historical track data collected, $93 \%, 5 \%$, and $2 \%$ of aircraft pairs would require 3,4 , and 5 mile wake turbulence minima, respectively. Therefore, for simplicity in this study, the 3-mile minimum representing $93 \%$ of aircraft pairs was assumed for all aircraft pairs. All route model nodes less than 40 miles from ORD modeled 3-mile separation, and all nodes 40 miles or more from ORD modeled 5-mile separation. A 0.3-mile separation buffer was added to these separation minima as a scheduling buffer. The separation requirements were converted from distance to time based on the nominal speeds associated with each node. Time ranges on both sides of reserved STAs of previously scheduled flights were blocked from consideration for new flight scheduling. The amount of time blocked on either side of each preexisting STA was based on the nominal speed of the flight that would be the follower. For example, if a previously scheduled flight $f_{1}$ had been assigned time $t$ at a given node, the blocked time range used for scheduling a new flight $f_{2}$ would be $[t$ $\left.\left(r / v_{1}\right), t+\left(r / v_{2}\right)\right]$ where $r$ is the required separation distance and $v_{1}$ and $v_{2}$ are the nominal speeds of $f_{1}$ and $f_{2}$, respectively. This way, if $f_{2}$ is scheduled in front of $f_{1}$, then $f_{1}$ is the follower and separation will be at least $r / v_{1}$, and if $f_{2}$ is scheduled behind $f_{1}$, then $f_{2}$ is the follower and separation will be at least $r / v_{2}$.

\section{Rescheduling due to tactical reconfiguration}

The first-come-first-served scheduler serves new flights in order of their entry point ETAs. However, in the case of a reconfiguration, many flights already scheduled and traveling assigned routes may need to be rescheduled and rerouted simultaneously. The scheduler is notified at time $t_{N}$ that a reconfiguration will occur at time $t_{R}$. All flights with originally scheduled runway arrival times greater than $t_{R}$ are rescheduled. Each flight is rescheduled by processing route options from the first originally scheduled node after $t_{N}$ to the new configuration runways, choosing the one with the earliest schedulable runway arrival time. Two methods of prioritizing the rescheduling of these flights were tested. Rescheduling in order of original scheduled runway arrival time prioritizes flights that were closer to landing and will likely fly extra distance at inefficiently low altitudes and speeds during the reconfiguration. Rescheduling in order of earliest estimated runway arrival time in the new configuration prioritizes packing the new schedule in an attempt to maintain throughput.

\section{Scenarios}

Arrival traffic scenarios for ORD were randomly generated in 15 minute chunks according to a given entry node distribution and quarter hourly rate. Each 15 minutes of arrival traffic contained the desired number of flights per quarter hour with uniform randomly distributed entry ETA across the 15 minutes. Random entry node assignment was weighted by the traffic representation percentages shown in Figure 2.

Historical ASPM data was analyzed to determine the arrival capacities of PX and WF configurations in current operations. In current operations, the most commonly reported quarter hourly arrival capacities are 24 for PX and 25 or 26 for WF. The actual arrivals per quarter hour are very similar for both configurations at higher traffic volumes 
with a mode throughput of 18 and 90th percentile throughputs similar to the capacities. The theoretical capacity for 3.3 miles separation at three runways with 130-knot nominal landing speed is 29 aircraft per quarter hour, but that is highly unlikely to be sustainable for extended periods of time. Therefore arrival traffic scenarios were created with quarter hourly rates ranging from 20 to 28 . A throughput of 28 falls within the $10 \%$ increase in throughput that precision scheduling could be expected to enable over the highest commonly observed capacity of 26 .

The total time a flight could be scheduled to spend within the route model (entry to runway) ranged between 18 and 30 minutes depending on the route option chosen and speed control delay applied. Therefore, to ensure that the route model was fully populated with flights at the time of reconfiguration notification, $t_{N}$ was set to 30 minutes from the start of the scenario. The reconfiguration time $t_{R}$ varied between 30 and 60 minutes after the start of the scenario in 5 minutes increments. Scenarios where $t_{R}=t_{N}$ were expected to be the most disruptive reconfigurations with no advanced notice. In today's operations, this would be similar to a missed approach triggering the decision to reconfigure. Scenarios where $t_{R}=60$ allowed 30 minutes lead-time for all flights occupying the route model at $t_{N}$ to land as originally scheduled, rescheduling only flights that had not yet entered the route model. Thus, the 30-minute lead time scenarios enter the realm of strategic reconfiguration.

A total of 100 traffic scenarios per arrival rate were generated. This number was chosen to generate fairly stable average results without making the simulation data collection time over the range of variables to large. For each traffic condition, reconfiguration scheduling was simulated for the range of reconfiguration lead-times for both PX to WF and WF to PX reconfiguration directions. Table 1 summarizes the range of scenario conditions simulated 100 times each.

Table 1. Scenario variables

\begin{tabular}{|l|l|l|l|l|}
\hline Variable & Min value & Max value & Increment & Total \\
\hline Reconfiguration direction & PX to WF & WF to PX & - & 2 \\
\hline Reschedule Priority & Original STA & New ETA & - & 2 \\
\hline Arrival Rate & 20 aircraft/qtr & 28 aircraft/qtr & 1 aircraft/qtr & 9 \\
\hline Reconfiguration lead-time $\left(t_{R}-t_{N}\right)$ & 0 min & 30 min & $5 \mathrm{~min}$ & 7 \\
\hline
\end{tabular}

\section{Metrics}

When a tactical or short notice reconfiguration occurs, the expected system impacts are temporary changes in throughput, and delay. Throughput represents the performance of the system as a whole, whereas delay can identify where the impact to the system was absorbed. In some simulations, a feasible reschedule solution cannot be found and the reconfiguration fails, which is a complexity indicator. No other metrics are collected for such simulations. Throughput and delay metrics were averaged across all successful simulations within the same variable subset.

1. Success Rate

The reschedule success rate is the percentage of 100 simulations within the same variable subset that succeeded and for which all other metrics were collected.

\section{Throughput}

Throughput is measured as the number of final STAs for a particular scheduling point per quarter hour. Airport arrival throughput was measured as a combination of each arrival runway throughput per quarter hour. Throughput differences per minute were computed between each reconfiguration simulation and the corresponding baseline simulation of the same traffic scenario without any reconfiguration.

3. Delay

Total delay is runway ETA minus STA. However delay can be absorbed prior to the entry point (Center delay) or between the entry point and runway (TRACON delay). Center delay is entry the point ETA minus the STA. TRACON delay can be further broken down into path delay or speed delay. Recall that the assigned path with the earliest runway STA may not be the path with the earliest runway ETA. This means that the nominal time-to-fly (TTF) of the assigned route is longer than the preferred shortest path TTF. Path delay is the difference between the assigned path TTF and the shortest path nominal TTF. Speed delay is the difference between the scheduled TTF and nominal TTF of the assigned path. For aircraft requiring rescheduling within the TRACON due to reconfiguration, the shortest path from the old configuration and assigned path from the new configuration are used in the delay calculations. In the case that the new configuration assigned path is shorter than the old configuration shortest path, path delay is negative. Delay metrics were averaged across all aircraft with runway STA in the same quarter hour. Delay differences per minute were computed between each reconfiguration simulation and the corresponding baseline simulation of the same traffic scenario without any reconfiguration. 


\section{Results}

\section{A. Success Rate}

The reschedule success rate was most dependent on the notification lead-time and arrival rate. No noteworthy difference was found between rescheduling prioritization methods or reconfiguration direction. Figure 6 shows the rescheduling success rate vs. arrival rate for each lead-time $\left(t_{R}-t_{N}\right)$. Each success rate represents 400 simulations (100 for each reconfiguration direction and rescheduling prioritization method combination).

The lowest and highest few lead-times were less sensitive to arrival rate. Almost no rescheduling attempts with 0 lead-time succeeded, whereas most or all rescheduling attempts with 20 minutes or greater lead-time succeeded. Lead-times between 5 and 15 minutes had higher success rates at low arrival rates. The success rates then degraded as the arrival rate increased.

A failed reschedule does not mean that no rescheduling solution exists. It only means that the heuristic firstcome-first-served rescheduling based on the given flight sequence according to priority method, reached a point where the next flight served had no fixed path or speed solution within $10 \%$ of nominal. In many cases, the same arrival rate scenario with the same leadtime succeeded for one prioritization method and failed for the other. The rescheduling prioritization by original runway STA accounted for a vast majority of the few successful reschedules with 0 lead-time. On the other hand, prioritization by new runway ETA had more success with lead-time between 5 and 15 minutes. Rescheduling success rate could be increased by applying a near-optimal rather than heuristic scheduling method, increasing path options, or expanding TTF bounds along path segments.

Based on the low rescheduling success rate of 0 lead-time simulations, metrics from the few 0 lead-time successes were not included in the remaining analyses.

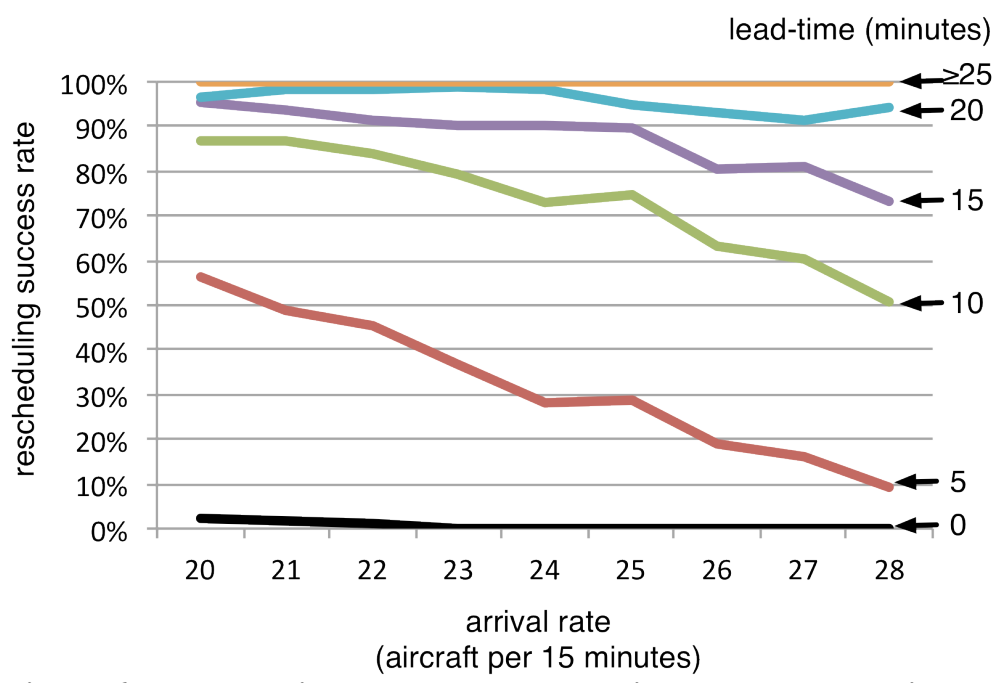

Figure 6. Rescheduling success rate vs. arrival rate per lead-time.

\section{B. Throughput}

A quarter hourly final runway STA throughput difference was calculated every minute and then smoothed with a 15 minute running average. Figure 7 shows the throughput difference results for each lead-time for one set of parameters (WF to PX, arrival rate $=27$, original STA rescheduling priority). This shows the typical throughput response to reconfiguration.

The 25- and 30-minute lead-time results hardly deviate from 0 (the 30 minute lead-time overlaps the 25 -minute curve), indicating that the reconfiguration had little to no effect on throughput. The other results have a

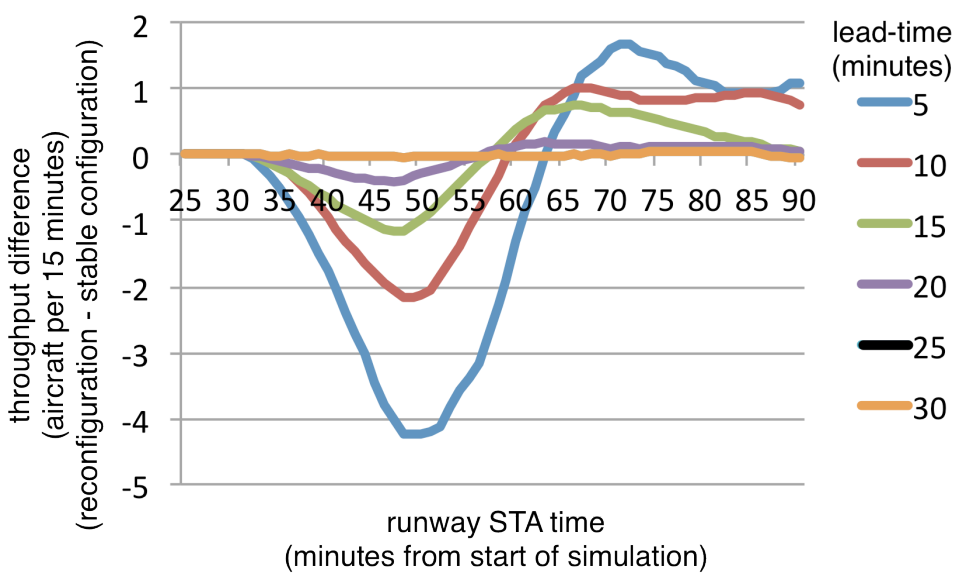

Figure 7. Sample throughput difference response per lead-time. period where the reconfiguration throughput is lower than that of the stable configuration, after which the reconfiguration throughput may be higher to recover from the recent throughput reduction and built up center delay. 
Figure 8 compares the peak throughput stress magnitude, which is the throughput difference when the reconfiguration drops below the stable configuration the most.

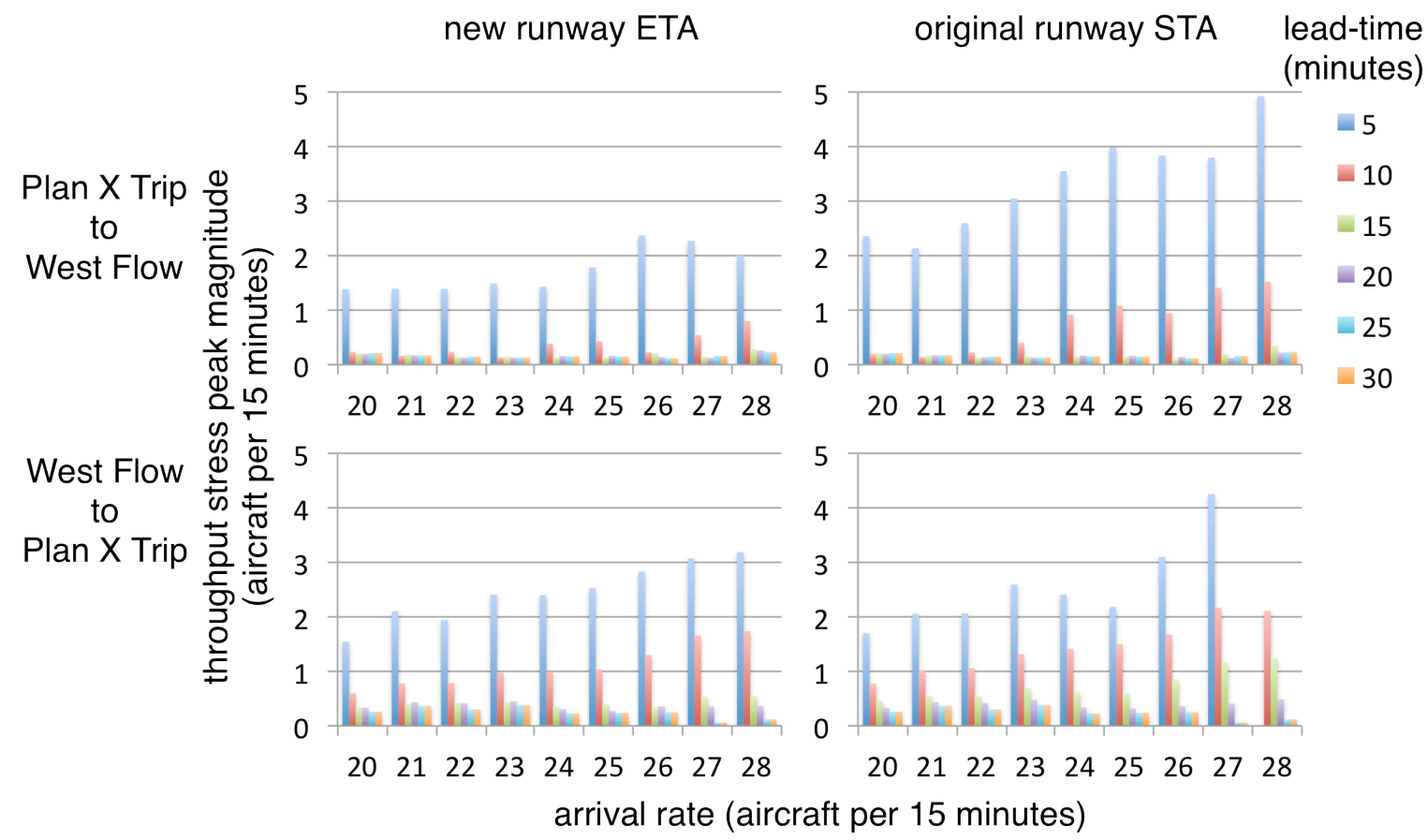

Figure 8. Throughout stress peak magnitude.

As expected, there is a general increase in throughput stress as arrival rate increases and lead-time decreases. However, there are also differences in magnitude between reconfiguration direction and rescheduling prioritization methods. Figure 9 shows the throughput stress magnitude difference between reconfiguration direction, and Fig. 10 shows the difference between rescheduling prioritization methods. With the exception of prioritizing by original runway STA with 5-minute lead-time, changing from WF to PX tends to stress throughput more than changing from PX to WF. This could be due to the complexity of transitioning from a 3-paralell runway configuration to 2-paralell plus 1-diagonal runway configuration. Rescheduling by the original runway STA tends to stress throughput more than rescheduling by the new runway ETA, especially when changing from PX to WF with lower lead-time. The throughput results suggest that in most cases, rescheduling prioritizing by new ETA is preferred over prioritizing by original STA. If the new ETA prioritization is used, 15 minute or greater lead-times will have a minimal impact on throughput at any arrival rate acceptable for stable configuration operations. Even at 10 minutes lead-time, the impact on throughput is low if the arrival rate is at or below 25 aircraft per quarter hour.

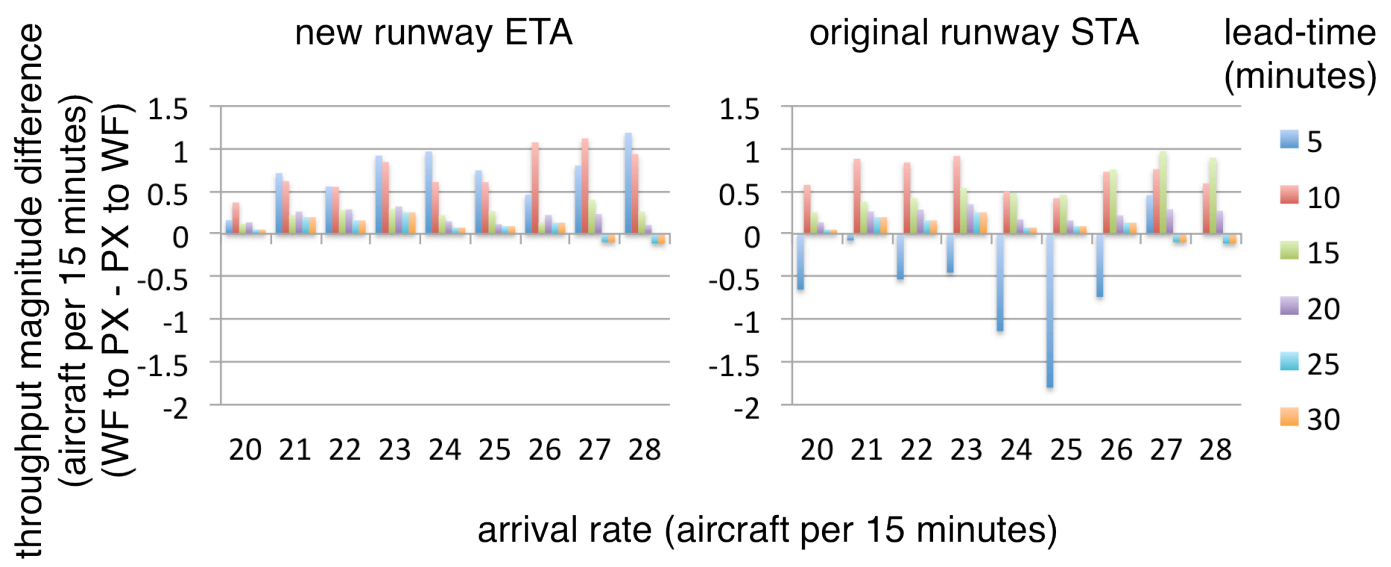

Figure 9. Throughput stress peak magnitude difference between reconfiguration direction. 


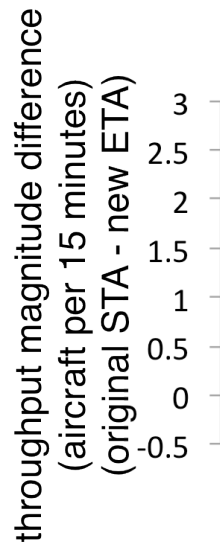

Plan X Trip to West Flow

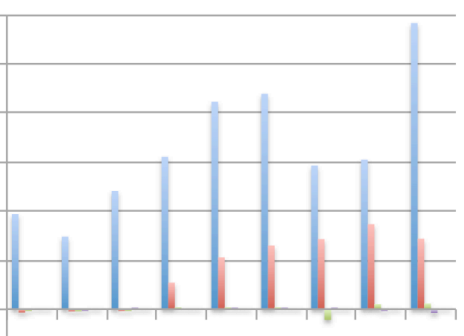

$202122 \quad 2324252627 \quad 28$

\section{West Flow to Plan X Trip}

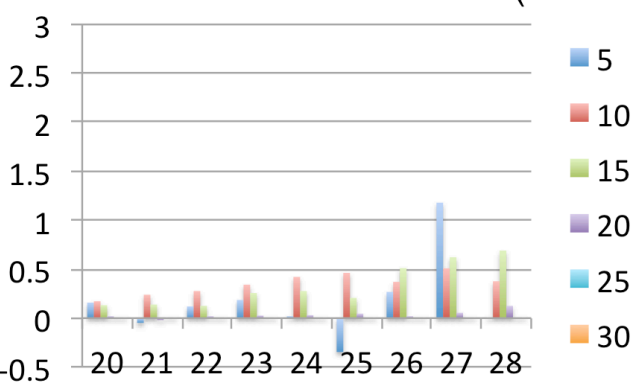

Figure 10. Throughput stress peak magnitude difference between rescheduling prioritization method.

\section{Delay}

As with throughput, quarter hourly average delay differences were calculated every minute and then smoothed with a 15 minute running average. Figure 11 compares center, path, speed, and total delay difference results for each lead-time between reconfiguration direction for a single set of remaining parameters (arrival rate $=25$ aircraft per quarter hour, original STA rescheduling priority).

Although at first glance the reconfiguration directions appear to have very different reactions to the reconfiguration, this is only because in stable configuration PX generates less average path and speed delay than WF. Therefore, the PX to WF reconfiguration transitions from a lower to higher average delay and the WF to PX reconfiguration transitions from a higher to lower average delay. In both cases, the peak delay difference magnitudes increase as reconfiguration lead-time decreases. The peaks appear in sequence: first speed, then path, and finally center delay. The negative speed delay peak results from shifting speed delay to path delay as flights rescheduled within the TRACON are assigned new paths. When path delay peaks and speed delay saturates, then additional delay
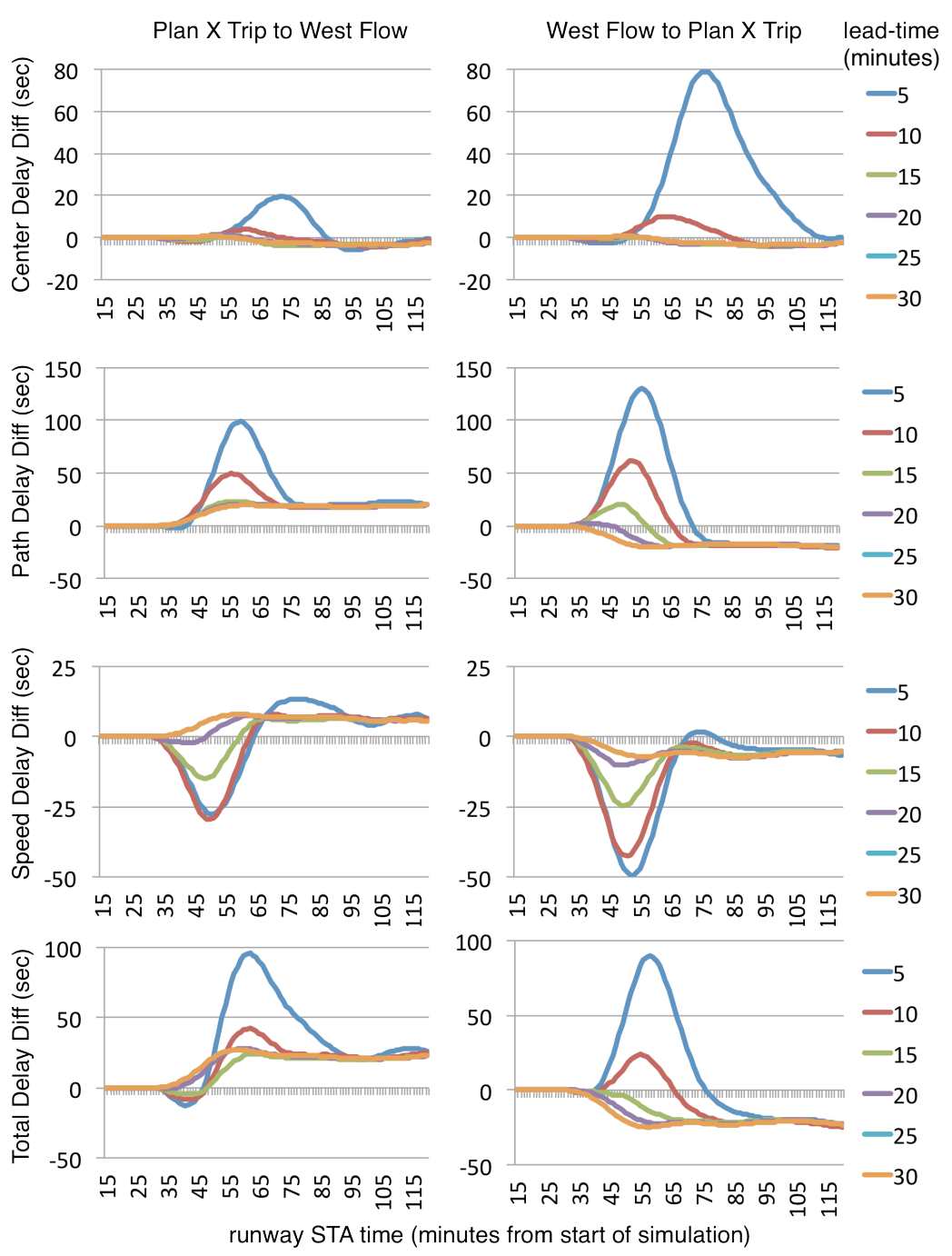

Figure 11. Delay comparison by reconfiguration direction. 
is passed to the center until natural gaps in the arrival demand return delay levels to what is normal for the new configuration. Because PX is not able to absorb as much path and speed delay, more delay is passed to the Center during the WF to PX reconfiguration. However, the total delay peaks are still similar between configuration change directions.

It is important to note that all 30 and 25-minute lead-time delay results achieved very smooth transitions with no transient response as they stabilized to the new configuration's nominal delay level. In this experiment these leadtimes resulted in strategic reconfigurations. The lead-time was great enough that flights needing rescheduling were always on a path common to both configurations, making the transition between configuration routing seamless. To focus on the more transient response associated with tactical rescheduling, the remaining lead-time results were compared and analyzed relative to the 30-minute lead-time results. Figure 12 shows the maximum deviations of 5 to 20 -minute lead-time total delay from the 30-minute lead-time total delay.

In figure 12, separate graphs are shown for each lead-time, and reconfiguration direction and rescheduling prioritization methods are compared within each graph. Keep in mind that results for high arrival rates at 5-minute lead-time may be skewed due to the very low success rate and therefore lack of data for these cases. For the 10 to 20-minute lead-times, WF to $\mathrm{PX}$ reconfigurations produce consistently higher peak average delay differences which is independent of arrival rate until 27 and 28 aircraft per quarter hour are reached. In general, there are higher peak average delays for original STA prioritization than new ETA prioritization. The difference becomes more pronounced as

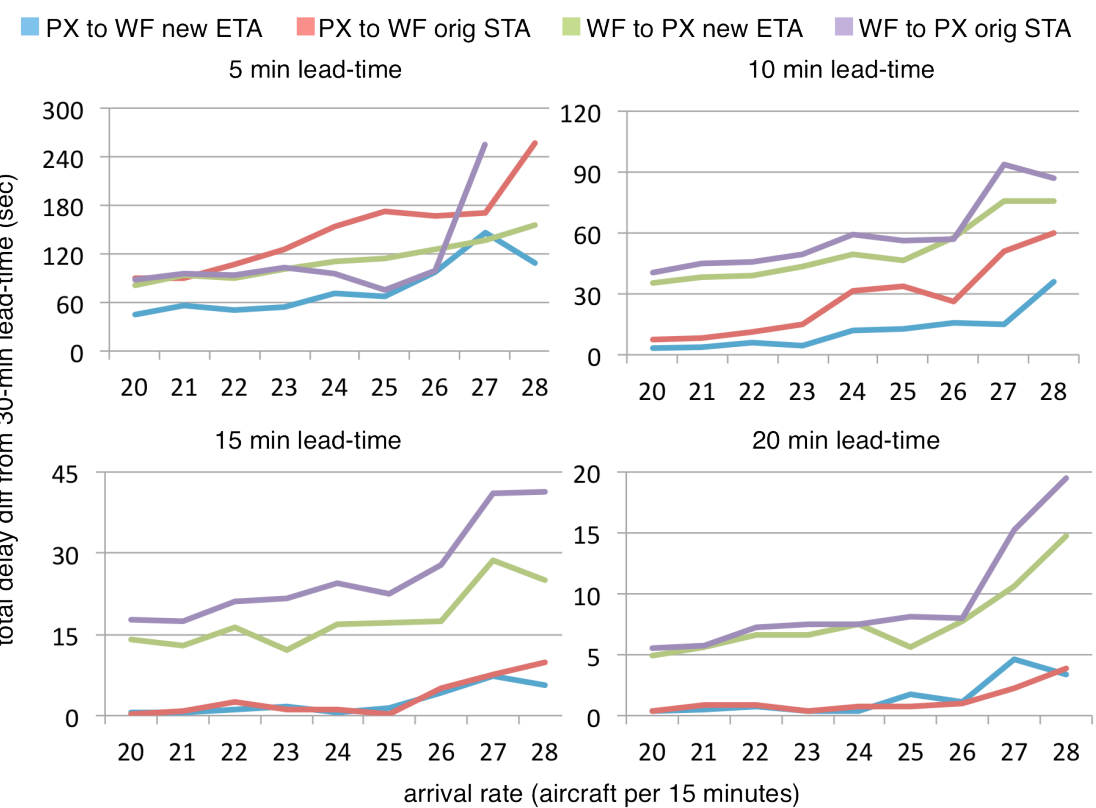

Figure 12. Total delay max deviation from 30-min lead-time results. the system is stressed with higher arrival rate and shorter lead-time.

Figure 13 shows the maximum deviations of delay from the 30-minute lead-time delay decomposed into center, path, and speed delay on the same scale. The 20-minute lead-time results are not included as they are too small to view in the same scale.

The impact of shorter lead-time on the delay response to reconfiguration amplifies for each type of delay as arrival rate increases. There is no significant speed delay difference between reconfiguration direction and rescheduling prioritization. On the other hand, for path delay, there is a very consistent difference between reconfiguration directions and a more subtle difference between rescheduling prioritization that amplifies as arrival rate increases. This is due to the difference in transition path options available to each reconfiguration. The center delay responses between reconfiguration direction and rescheduling prioritization tend to deviate from one another as arrival rate increases and lead-time decreases. This is a reflection of both the reconfiguration direction's capacity for path and speed delay, and the total delay impact of the rescheduling prioritization method.

The delay results support the throughput analysis conclusions in that prioritizing by new ETA is preferred over prioritizing by original STA. The delay results also suggest that the reason there is little impact to throughput with lead-times of 10 minutes or greater is that the path and speed delay capacity of the reconfiguration transition routing at this lead-time is large enough that very little delay is passed to the center. Once center delay starts to build, throughput starts to become heavily impacted. Even though throughput may be robust, the 10-20 minutes lead-times still have a negative impact on individual flight efficiency. The shorter the lead-time, the longer aircraft are forced to fly at low speeds due to the constraint that flights may only reduce speed and limitation on transition routing available. 


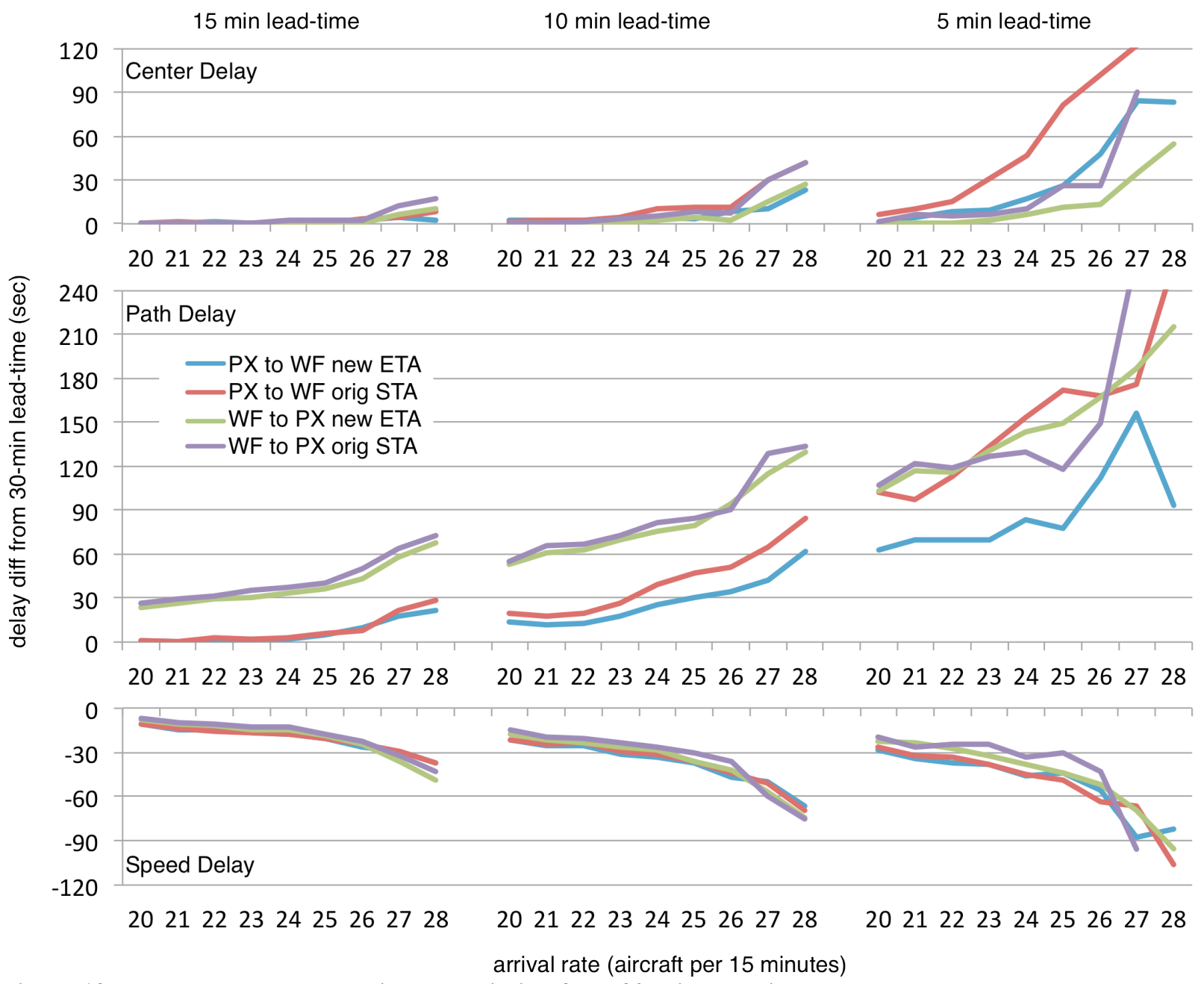

Figure 13. Decomposed delay maximum deviation from 30-min lead-time results.

\section{Conclusion}

This work developed a model of fixed path routing for reconfiguration between ORD's top two peak traffic configurations. A first-come-first-served multi-point scheduler was adapted to the reconfiguration problem by prioritizing the rescheduling of aircraft within the TRACON at the time of reconfiguration notification.

Results suggest that a rescheduling prioritization based on earliest ETA in the new configuration is preferred over prioritization based on the original STA assigned in the old configuration. Neither method was $100 \%$ successful in rescheduling below 25 minutes lead-time, especially at higher arrival rates. A relaxation of the separation constraints (temporarily smaller scheduling buffer) or increase in speed control range or path options could increase the success rate.

From a system impact perspective, reconfigurations with lead-times as short as 10 minutes at a nominal stable configuration arrival rate ( $\sim 25$ arrivals per quarter hour) could be accommodated with little impact to throughput. As lead-time shortened below 25-minutes, individual aircraft efficiency quickly degraded due to extra flight time at lower speeds.

The reconfiguration generally had less impact in one reconfiguration direction than another due to the inherently different and asymmetrical route designs. Changing from the 3-parallel-runway configuration to the 2-parallel-plus1-diagonal-runway configuration was more difficult than visa versa.

This work demonstrates that first-come-first-served arrival scheduling of fixed routing for tactical reconfiguration is promising if at least 10-15 minutes lead-time is given before the reconfiguration is in effect. More thought must be given to transition routing design if this scheduling approach is to accommodate shorter lead-times.

12

American Institute of Aeronautics and Astronautics 


\section{Acknowledgments}

This work was funded by the Concept and Technology Development Project, which is part of NASA's Airspace Systems Program.

\section{References}

${ }^{1}$ Hunter, G., "Empirical Evaluation of Impact of Reconfiguration Events on Airport Capacity," $12^{\text {th }}$ AIAA Aviation Technology, Integration, and optimization (ATIO) Conference and $14^{\text {th }}$ AIAA/ISSM, AIAA-2012-4434, Indianapolis, Indiana, 2012.

${ }^{2}$ Roach, K., "Procedures for Changing the Flow Direction at Dallas/Fort Worth International Airport," NASA North Texas Research Station (NTX), Fort Worth, Texas, 2011 (Unpublished, for access contact the author at keenan.r.roach@nasa.gov).

${ }^{3}$ Roach, K., "Procedures for Changing the Flow Direction at Chicago O'Hare International Airport," NASA North Texas Research Station (NTX), Fort Worth, Texas, 2013 (Unpublished, for access contact the author at keenan.r.roach@nasa.gov).

${ }^{4}$ Oseguera-Lohr, R., N. Phojanamongkolkij, G. Lohr, and J. Fenbert, "Benefits Assessment for Tactical Runway Configuration Management Tool," $13^{\text {th }}$ AIAA Aviation Technology, Integration, and optimization (ATIO) Conference, AIAA2013-4395, Los Angeles, California, 2013.

${ }^{5}$ Bai, X. and P.K. Menon, "Decision Support for Optimal Runway Reconfiguration," $13^{\text {th }}$ AIAA Aviation Technology, Integration, and optimization (ATIO) Conference, AIAA-2013-4397, Los Angeles, California, 2013.

${ }^{6}$ Zhang, R. and R. Kincaid, "Robust Optimization Model for Runway Configurations Management," $11^{\text {th }}$ AIAA Aviation Technology, Integration, and optimization (ATIO) Conference, AIAA-2011-6922, Virginia Beach, Virginia, 2011.

${ }^{7}$ Tandale, M., S. Vaddi, S. Wiraatmadja, and V. Cheng, "A Queuing Framework for Terminal Area Operations," AIAA Guidance, Navigation, and Control Conference, AIAA-2011-6363, Portland, Oregon, 2011.

${ }^{8}$ Swenson, H., J. Thipphavong, A. Sadovsky, L. Chen, C. Sullivan, and L. Martin, "Design and evaluation of the terminal area precision scheduling and spacing system," 9th USA/Europe Air Traffic Management R\&D Seminar, FAA and EUROCONTROL, Berlin, Germany, 2011.

${ }^{9}$ Callantine, T. J., C. Cabrall, M. Kupfer, L. Martin, J. Mercer, E. A. Palmer, "Investigating the impact of off-nominal events on high-density 'green' arrivals," 30th Digital Avionics Systems Conference, IEEE, Seattle, Washington, 2011.

${ }^{10}$ Swenson, H., J. Jung, J. Thipphavong, L. Chen, L. Martin, and J. Nguyen, "Development and evaluation of the terminal precision scheduling and spacing system for off-nominal condition operations," 31st Digital Avionics Systems Conference, IEEE, Williamsburg, Virginia, 2012.

${ }^{11}$ Federal Aviation Administration, Aviation System Performance Metrics (ASPM) Airport Efficiency, URL: http://aspm.faa.gov [cited 7 January 2013].

${ }^{12}$ Zelinski, S., “A Graph-Based Approach to Defining Nominal Terminal Routing,” 31st Digital Avionics System Conference, IEEE, Williamsburg, Virginia, 2012.

${ }^{13}$ Meyn, L., "A Closed-Form Solution to Multi-Point Scheduling Problems," AIAA Modeling and Simulation Technologies Conference, AIAA, Toronto, Ontario Canada, 2010.

${ }^{14}$ Federal Aviation Administration, 2012, Air Traffic Control FAA Order JO 7110.65U Chapter 5. Section 5. Radar Separation, URL: http://www.faa.gov [cited 11 May 2012]. 